\title{
Performance improvement of high thickness photopolymers for holographic data storage applications
}

\author{
M. Ortuño ${ }^{1,2, *}$, S. Gallego ${ }^{1,2}$, A. Márquez ${ }^{1,2}$, C. Neipp ${ }^{1,2}$, I. Pascual ${ }^{2,3}$, A. Beléndez ${ }^{1,2}$ \\ ${ }^{1}$ Dpto. Física, Ing. Sistemas y T ${ }^{\text {a }}$ de la Señal, Universidad de Alicante. \\ ${ }^{2}$ Instituto Universitario de Física Aplicada a las Ciencias y las Tecnologías, Universidad de Alicante. \\ ${ }^{3}$ Dpto. de Óptica, Farmacología y Anatomía, Universidad de Alicante, Apdo. 99, 03080 Spain \\ *mos@ua.es
}

\begin{abstract}
We use 4,4' azo-bis-(4-cyanopentanoic acid) as chain transfer agent in a photopolymer with triethanolamine/yellowish eosin as initiator system. It is possible to work in a particular conditions to get a chain transfer effect minimizing the decomposition of 4,4' azo-bis-(4-cyanopentanoic acid) by the sensitized dye. The improved photopolymer has a low scattering due to the low molecular weight of the generated polymer chains. This is related to the chain transfer effect in the size of the polymer chains. It is important to establish the optimum concentration of chain transfer agent to avoid decreasing the maximum diffraction efficiency due to a low molecular weight of the polymer chains.
\end{abstract}

\section{INTRODUCTION}

Photopolymers with a hydrophilic matrix such as polyvinyl alcohol PVA are versatile holographic recording materials for use in hologram recording experiments. They use water as the solvent and can be made in layers of different thicknesses [1-3]. One of the photopolymers most widely used is composed of acrylamide AA, as polymerizable monomer, triethanolamine TEA, as radical initiator, yellowish eosin YE, as dye and PVA and water as binder. TEA is the initiator most often used with dyes derived from fluorescein because they can generate a radical by a redox reaction under excitation by light. The dye is bleached in this reaction because it is decomposed in the photoinitiation reaction [4,5-7].

The use of a redox initiation is not possible for other compounds such as pyrromethene derived dyes because they are not photoreducible and thus another initiation system is necessary in these cases. 4,4' azo-bis-(4-cyanopentanoic acid) ACPA, is a water soluble radical initiator used in polymerizations in solution with thermal initiation. The molecule produces two radicals and one nitrogen molecule when it reacts with the excited dye in the initiation and the radicals generated react with the monomer in the propagation step. We used this initiator with a dye derived from pyrromethene in a solid layer polymerization and we developed in a previous study a holographic recording material that contains ACPA and pyrromethene dye in a PVA matrix. We saw that ACPA is an interesting initiator for this kind of dyes which can not react with TEA under a redox reaction [8].

In this paper, we use ACPA as a chain transfer agent in a photopolymer due to its capacity to generate two identical radicals. TEA/YE is the redox system with TEA as initiator. Therefore, it is necessary to check the conditions of the chain transfer experiments to minimize the initiation capacity of the ACPA molecule.

When a diffraction grating is recorded in a photopolymer, a radical polymerization initiated by light takes place in the exposed zones. The basic mechanism in a radical polymerization involves the following reactions [9]:

Smart Nano-Micro Materials and Devices, edited by Saulius Juodkazis, Min Gu, Proc. of SPIE

Vol. 8204, 820432 - @ 2011 SPIE · CCC code: 0277-786X/11/\$18 · doi: 10.1117/12.902889 
Initiation

$$
\begin{aligned}
& \mathrm{I} \stackrel{\mathrm{k}_{\mathrm{d}}}{\longrightarrow} 2 \mathrm{R} \cdot \\
& \mathrm{R} \cdot+\mathrm{M} \stackrel{\mathrm{k}_{\mathrm{i}}}{\rightarrow} \mathrm{M}_{1} \cdot \\
& \mathrm{M}_{1} \cdot+\mathrm{M}^{\text {Propagation }} \stackrel{\mathrm{k}_{\mathrm{p}}}{\rightarrow} \mathrm{M}_{2} \cdot \\
& \mathrm{M}_{2} \cdot+\mathrm{M} \stackrel{\mathrm{k}_{\mathrm{p}}}{\rightarrow} \mathrm{M}_{3} \cdot \\
& \mathrm{M}_{\mathrm{n}} \cdot+\mathrm{M} \stackrel{\mathrm{k}_{\mathrm{p}}}{\rightarrow} \mathrm{M}_{\mathrm{n}+1} \cdot \\
& \mathrm{M}_{\mathrm{x}} \cdot+\mathrm{M}_{\mathrm{y}} \cdot \stackrel{\mathrm{k}_{\mathrm{tc}}}{\longrightarrow} \mathrm{M}_{\mathrm{x}+\mathrm{y}} \\
& \mathrm{M}_{\mathrm{x}} \cdot+\mathrm{M}_{\mathrm{y}} \cdot \stackrel{\mathrm{k}_{\mathrm{td}}}{\longrightarrow} \mathrm{M}_{\mathrm{x}}+\mathrm{M}_{\mathrm{y}}
\end{aligned}
$$

The radicals $\mathrm{R} \bullet$ derived from the initiator molecule (TEA) react with the monomer M (AA) generating a growing macroradical $\mathrm{M} \bullet$ in the propagation reaction system. Two growing macroradicals are inactivated by combination or deproportion in the termination reactions. The kinetic constants are denoted by the letter ' $\mathrm{k}$ ' and a subscript for each reaction. In a photopolymerization, the mechanism of initiation is more complex but in this work we will centre on the propagation. A more detailed scheme is included in Ref. [7].

\subsection{Chain transfer mechanism}

The incorporation of a chain transfer agent XA into the photopolymer introduces a new reaction in the propagation reaction system of the kinetic scheme:

$$
M_{n} \cdot+X A \stackrel{k_{t r}}{\longrightarrow} M_{n}-X+A \cdot
$$

Where Mn• is a growing macroradical that reacts with the chain transfer agent XA producing deactivation of the growing macroradical. The new radical A• can start a new polymer chain according to the following reaction:

$$
A \cdot+M \stackrel{k_{a}}{\longrightarrow} M \cdot
$$

The main effect of the chain transfer is the decrease in the polymer chain molecular weight due to the presence of XA molecules which deactivate the growing macroradicals Mn•.

We use ACPA as chain transfer agent XA with TEA/YE as redox initiator system. We analyze the influence of the chain transfer effect on the holographic characteristics of the photopolymer.

\section{HOLOGRAPHIC SET-UP}

The holographic set-up is shown in Figure 1. An Argon laser at a wavelength of $514 \mathrm{~nm}$ was used to store diffraction gratings by means of continuous laser exposure. The laser beam was split into two secondary beams with an intensity ratio of 1:1. The diameter of these beams was increased to $1.5 \mathrm{~cm}$ with an expander, while spatial filtering was ensured. The object and reference beams were recombined at the sample at an angle $\theta$ ( $16.8^{\circ}$ to the normal) with an appropriate 
set of mirrors, and the spatial frequency obtained was 1125 lines $/ \mathrm{mm}$. The diffracted and transmitted intensity were monitored in real time with a He-Ne laser positioned at Bragg's angle $\left(\theta^{\prime}=20.8^{\circ}\right)$ tuned to $633 \mathrm{~nm}$, where the material is not sensitive. The diffraction efficiency (DE) was calculated as the ratio of the diffracted beam to the incident power. The transmission efficiency (TE) was calculated as the ratio of the transmitted beam to the incident power. We record unslanted gratings in a photopolymer layer using a low recording light intensity of $5 \mathrm{~mW} / \mathrm{cm}^{2}$ to prevent the ACPA molecule from acting as initiator.

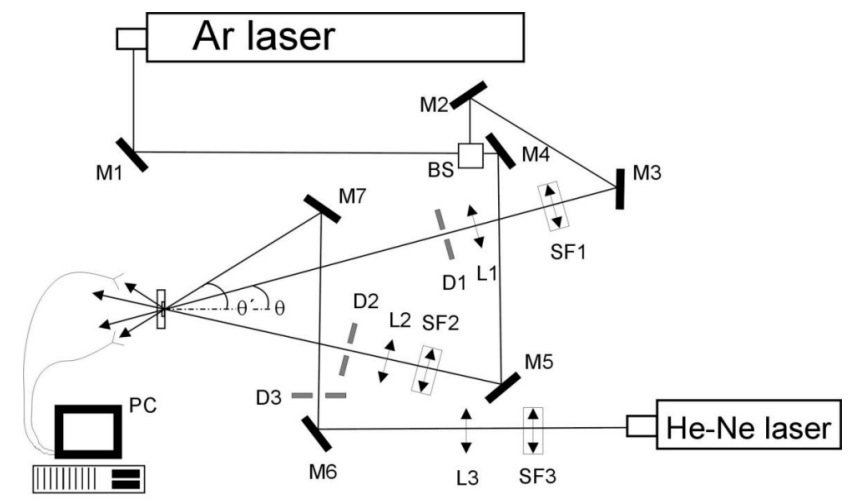

Figure 1. Experimental set-up: BS, beamsplitter, Mi, mirror, SFi, spatial filter, Li, lens, Di, diaphragm, PC, data recorder.

\section{PREPARATION OF THE MATERIAL}

In holographic recording materials based on PVA/AA, a solution of PVA in water forms the matrix and this is used to prepare the solution of monomer AA, chain transfer agent ACPA and the photopolymerization initiator system: yellowish eosin and triethanolamine. PVA and ACPA were supplied by Fluka, AA and TEA by Sigma and YE by Panreac.

We prepare the solutions using a conventional magnetic stirrer, under red light and left in a climatic chamber (Fisher scientific climacell 222) to allow the water to evaporate in controlled conditions $\left(\mathrm{T}=20 \pm 1{ }^{\circ} \mathrm{C}\right.$, relative humidity= $45 \pm 5 \%$ ). The concentrations of the components in the optimized photopolymer solution can be seen in Table 1 . The solution is deposited in order to obtain solid layers of recording media [3,10]. The solutions are deposited by gravity in circular polystyrene molds to avoid strain and distortion in the material during the water evaporation process. The "dry" material is removed from the mold, cut into squares and adhered, without adhesive, to the surface of glass plates measuring $6.5 \times 6.5 \mathrm{~cm}^{2}$. The plates are then ready for exposure, which takes place immediately. The thickness of the material is measured using a micrometer with a sensitivity of $10 \mu \mathrm{m}$. Previous studies show that the quality of the films is high $[4,5,10]$.

Table 1. Component concentrations in the prepolymer syrup.

\begin{tabular}{lcc}
\multicolumn{1}{c}{ component } & photopolymer & photopolymer \\
& $\mathrm{A}$ & $\mathrm{B}$ \\
AA & $0.34 \mathrm{M}$ & $0.34 \mathrm{M}$ \\
TEA & $0.15 \mathrm{M}$ & $0.15 \mathrm{M}$ \\
YE & $9.1 \times 10^{-5} \mathrm{M}$ & $9.1 \times 10^{-5} \mathrm{M}$ \\
ACPA & $4.9 \times 10^{-3} \mathrm{M}$ & - \\
PVA Fluka 18- & $13.4 \% \mathrm{~m} / \mathrm{v}$ & $13.4 \% \mathrm{~m} / \mathrm{v}$ \\
88 & & \\
$\mathrm{M}_{\mathrm{w}}=130000 \mathrm{u}$ & &
\end{tabular}




\section{RESULTS}

We prepare photopolymer layers with the concentrations included in Table 1. The material has the YE/TEA initiator system and ACPA as chain transfer agent. We record an unslanted diffraction grating in the material at $5 \mathrm{~mW} / \mathrm{cm}^{2}$.

The influence of the chain transfer effect on the holographic scattering is analyzed in Figure 2 which shows the diffraction efficiency plus transmission efficiency versus energetic exposure for both photopolymers. A high value of $\mathrm{DE}+\mathrm{TE}$ is directly related to a low scattering.

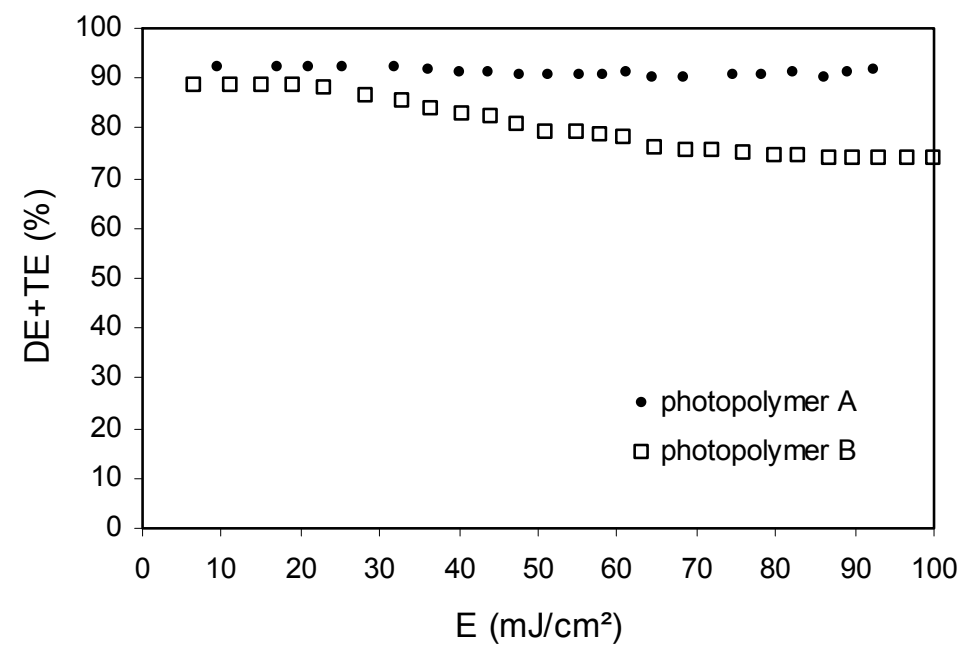

Figure 2. Diffraction efficiency and transmission efficiency versus energetic exposure.

During the recording, the sum $\mathrm{DE}+\mathrm{TE}=90-92 \%$ for the photopolymer with ACPA (photopolymer A) and DE $+\mathrm{TE}=74-$ $89 \%$ for the photopolymer B. This result indicates that the photopolymer with ACPA has a low scattering, and this implies a low polymer chain molecular weight because scattering is related to the size of the polymer chains. This affirmation is based on two considerations that we will now explain.

A low scattering implies a high value for coefficient $\Gamma$ in Kogelnik's coupled wave equation 1 and therefore higher $\mathrm{DE}+\mathrm{TE}$ values during the recording.

$$
\mathrm{DE}=\frac{\mathrm{I}_{\mathrm{D}}}{\mathrm{I}_{0}}=\Gamma \sin ^{2} \frac{\pi \mathrm{n}_{1}(\mathrm{t}) \mathrm{d}}{\lambda^{\prime} \cos \theta_{\mathrm{i}}{ }^{\prime}}
$$

In this equation $\Gamma$ is the absorption, diffusion and reflection losses factor, related to scattering. The presence of ACPA inside the photopolymer affects the light diffusion losses but the absorption and reflection losses are approximately constant.

$\theta_{\mathrm{i}}^{\prime}$ is the reconstruction beam angle (Figure 1), measured in the material, which is calculated using Snell's law. $\lambda^{\prime}$ is the reconstruction beam wavelength. $\mathrm{n}_{1}(\mathrm{t})$ is the refraction index modulation and $\mathrm{d}$ is the diffraction grating thickness $[11,12]$.

The light diffused by a polymer solution with a molecular weight $\mathrm{M}_{\mathrm{w}}$ can be expressed by equation 2, where: polymer concentration (c), light diffusion angle $(\theta)$, solution refraction index (n), incident light wavelength $\left(\lambda_{0}\right)$, polymer chains mean quadratic radius $\left(\mathrm{R}_{\mathrm{G}}{ }^{2}\right)$, system optics characteristics constant $(\mathrm{K})$ and $\mathrm{A}_{2}, \mathrm{~A}_{3}, \ldots$ are the virial coefficients $[13,14]$. 
The polymer Rayleigh relation $\left(\Delta \mathrm{R}_{\theta}\right)$ is proportional to the diffused light at angle $\theta$ divided by incident light. Polymer chains with a high $\mathrm{M}_{\mathrm{w}}$ have a high $\Delta \mathrm{R}_{\theta}$ and thus the light diffusion is also high [15].

$$
\frac{\mathrm{Kc}\left(1+\cos ^{2} \theta\right)}{\Delta \mathrm{R}_{\theta}}=\frac{1}{\mathrm{M}_{\mathrm{w}}}+\frac{16 \pi^{2} \mathrm{n}^{2}}{3 \lambda_{0}^{2} \mathrm{M}_{\mathrm{w}}} \mathrm{R}_{\mathrm{G}}^{2} \operatorname{Sen}^{2} \frac{\theta}{2}+2 \mathrm{~A}_{2} \mathrm{c}+3 \mathrm{~A}_{3} \mathrm{c}^{2}+\ldots
$$

Figure 3 shows the diffraction efficiency versus angular scan for photopolymer layers with different ACPA concentrations: $0 \mathrm{M}(910 \pm 10 \mu \mathrm{m}), 4.9 \times 10^{-3} \mathrm{M}(900 \pm 10 \mu \mathrm{m})$ and $9.0 \times 10^{-3} \mathrm{M}(900 \pm 10 \mu \mathrm{m})$. The graphs are obtained after the recording process.

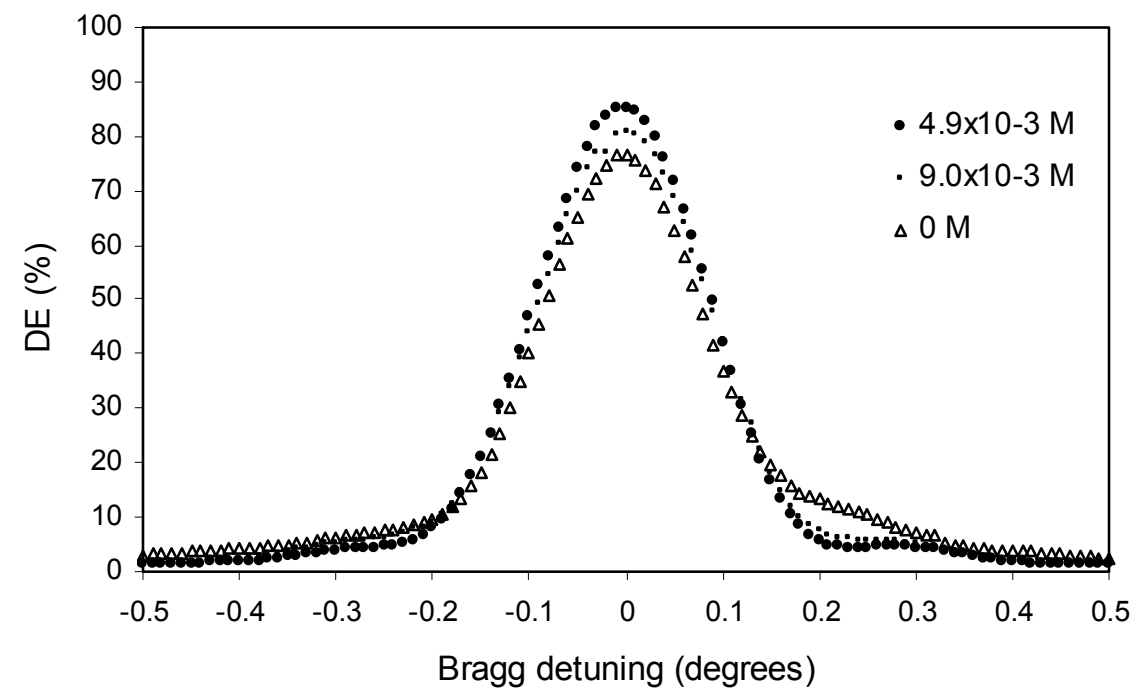

Figure 3. DE variation with the ACPA concentration.

The maximum diffraction efficiency DEmax is higher than 85\% without Fresnel correction for the photopolymer with $\mathrm{C}_{\mathrm{ACPA}}=4.9 \times 10^{-3} \mathrm{M}$. The low light losses obtained with this photopolymer (Figure 5) enable us to obtain a maximum diffraction efficiency higher than that of the standard photopolymer without ACPA (DEmax= 76\%).

If the ACPA concentration is increased $\left(9.0 \times 10^{-3} \mathrm{M}\right)$, a slightly lower maximum diffraction efficiency is obtained (DEmax $=81 \%$ ). When the chain transfer effect is enhanced the polymer chains generated in the photopolymerization have a very low molecular weight, their capacity for index refraction modulation is decreased (the refraction index is minor at the exposed zones of the diffraction grating) and the DEmax value is decreased according to Kogelnik's coupled wave equation 1. Therefore, it is very important to establish the optimum ACPA concentration (the chain transfer effect) to avoid a decrease in the DEmax value due to a very high concentration of ACPA.

\section{CONCLUSION}

The use of ACPA with low recording intensity and exposure lets to obtain a remarkably high value for the diffraction efficiency (DEmax $>85 \%$ ). Furthermore, it provides a remarkable high value for the sum of diffraction efficiency and transmission efficiency ( $\mathrm{DE}+\mathrm{TE}=90 \%$ ) with a very small variation of $2 \%$ during the recording. The differences obtained 
are very significant: for the standard photopolymer we obtain DEmax $<80 \%$ and a DE+TE variation $>10 \%$ during recording. Moreover, to our knowledge it is the first time that a diffraction efficiency higher than $80 \%$ has been obtained for this type of photopolymer with a thickness over $500 \mu \mathrm{m}$.

The results obtained are related to the effect induced by ACPA and its influence in the size of polymer chains. Moreover, we think that not only the light losses are implied in these results. The photopolymer with ACPA has a high fraction of low molecular weight polymer chains. These relatively small molecules could define better the three-dimensional sinusoidal contour of the fringes in the diffraction grating. This results in an improvement in the visibility of the fringes and therefore a low scattering and a high diffraction efficiency could be obtained. This effect could be more important when the spatial period of the fringes is similar to the size of the polymer chains generated in the photopolymerization [16].

\section{ACKNOWLEDGMENTS}

This work was supported by "Ministerio de Ciencia e Innovación”, Spain (FIS2008-05856-C02-01, FIS2008-05856-C02-02) and by “Generalitat Valenciana”, Spain (PROMETEO 2011/021).

\section{REFERENCES}

[1] R. A. Lessard, "Polymer used as holographic recording materials: a review," Proc. SPIE 3227, 199 (1997).

[2] M. Ortuño, E. Fernández, S. Gallego, A. Beléndez, and I. Pascual, "New photopolymer holographic recording material with sustainable design,” Opt. Express 15, 12425-12435 (2007).

[3] E. Fernández, M. Ortuño, S. Gallego, Andrés Márquez, C. Garcia, R. Fuentes, A. Beléndez, I. Pascual, "Optimization of a holographic memory setup using an LCD and a PVA-based photopolymer," Optik 121, 151158 (2010).

[4] M. Ortuño, S. Gallego, C. García, I. Pascual, C. Neipp, and A. Beléndez, "Holographic characteristics of an acrylamide/bisacrylamide photopolymer in 40-1000 $\mu \mathrm{m}$ thick layers,” Phys. Scr. T118, 66-68 (2005).

[5] M. Ortuño, S. Gallego, C. García, C. Neipp, and I. Pascual, "Holographic characteristics of a 1 mm thick photopolymer to be used in holographic memories,” Appl. Opt. 42, 7008-7012 (2003).

[6] G. Manivannan, P. Leclere, S. Semal, R. Changkakoti, Y. Renotte, Y. Lion and R. A. Lessard, "Photobleaching of xanthene dyes in a poly(vinyl alcohol) matrix,” Appl. Phys. B 58, $73-77$ (1994).

[7] L. Carretero, S. Blaya, R. Mallavia, R. Fernando Madrigal, A. Beléndez and A. Fimia, "Theoretical and experimental study of the bleaching of a dye in a film-polymerization process,” Appl. Opt. 37, 4496-4499 (1998).

[8] M. Ortuño, A. Márquez, S. Gallego, C. Neipp, E. Fernández, "Pyrromethene dye and non-redox initiator system in a hydrophilic binder photopolymer,” Opt. Mater. 30, 227-230 (2007).

[9] G. Odian, Principles of Polymerization, John Wiley \& Sons, Inc. New Jersey, USA (2004).

[10] M. Ortuño, S. Gallego, C. García, C. Neipp, A. Beléndez, and I. Pascual, "Optimization of a 1 mm thick PVA/acrylamide recording material to obtain holographic memories: method of preparation and holographic properties,” Appl. Phys. B 76, 851-857 (2003).

[11] H. Kogelnik, “Coupled Wave Theory for Thick Hologram Gratings,” Bell Sys. Tech. J. 48, 2909-2947 (1969).

[12] S. Gallego, M. Ortuño, C. Neipp, A. Márquez, A. Beléndez, I. Pascual, J. Kelly, and J. Sheridan, "Physical and effective optical thickness of holographic diffraction gratings recorded in photopolymers," Opt. Express 13, 1939-1947 (2005).

[13] I. Katime, and J. R. Quintana, [Scattering properties: light and X-rays in Comprehensive polymer science vol. 1], Pergamon press, Oxford, 103-132 (1989).

[14] B. Chu, [Laser light scattering], Academic Press, New York (1974).

[15] M. Ortuño, C. Neipp, S. Gallego, and A. Beléndez, "Linear response deviations during recording of diffraction gratings in photopolymers,” Opt. Express 17, 13193-13201 (2009).

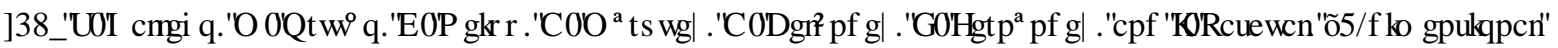

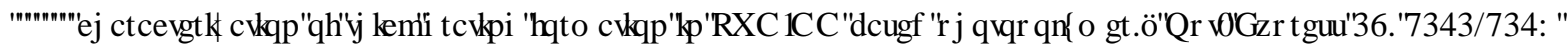

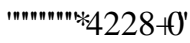

